\title{
A Defect in Tryptophan Metabolism
}

\author{
PAUL W. K. WONG, ${ }^{(25)}$ PHILLIP FORMAN, BORIS TABAHOFF, AND PARVIN JUSTICE
}

Department of Pediatrics, Abraham Lincoln School of Medicine, University of Illinois, Chicago, Illinois, USA

\section{Extract}

Oral tryptophan loading tests were performed in a patient with photosensitive pellagra-like skin rash and cerebellar ataxia but without hyperaminoaciduria. Plasma tryptophan concentrations after loading were similar in the patient and control subjects. Average urinary excretion of tryptophan in the patient from 0 to 6 and 6 to $12 \mathrm{hr}$ was 2.69 and $2.58 \mu \mathrm{mol} / \mathrm{kg}$, respectively; that in the control subjects was 0.82 and $0.34 \mu \mathrm{mol} / \mathrm{kg}$, respectively. However, the average renal clearance of tryptophan during the first $6 \mathrm{hr}$ of the loading tests in the patient was $0.757 \mathrm{ml}$ plasma $/ 1.73 \mathrm{~m}^{2}$ and that in the control subjects was $0.706 \mathrm{ml}$ plasma/ $1.73 \mathrm{~m}^{2}$.

Renal excretion of kynurenine in the patient was markedly decreased. The average from 0 to 6 and 6 to $12 \mathrm{hr}$ in the patient was 1.90 and $1.13 \mu \mathrm{mol} / \mathrm{kg}$, respectively; that in the control subjects was 12.90 and $18.15 \mu \mathrm{mol} / \mathrm{kg}$, respectively. Under ultraviolet light, paper chromatograms of urine from the patient showed a deficiency of xanthurenic acid, kynurenic acid, kynurenine, and formylkynurenine. The deficiency of formylkynurenine in the patient's urine was confirmed by staining the paper chromatograms with Ehrlich's reagent.

The patient was "sensitive" to oral nicotinic acid treatment; however, oral nicotinamide was well tolerated with improvement in the photosensitive skin rash.

\section{Speculation}

The normal absorption of tryptophan and the marked decrease of formylkynurenine and kynurenine in the urine after tryptophan loading in this patient suggest a metabolic block in tryptophan oxidation. The lack of formylkynurenine excretion suggests a deficiency of tryptophan pyrrolase (L-tryptophan 2,3-dioxygenase).

Hartnup disease, first described by Baron et al. (2), and congenital tryptophanuria, reported by Tada et al. (16), are characterized clinically by intermittent cerebellar ataxia and photosensitive, pellagra-like skin rash. Milne and his associates (9) produced evidence that there was an impairment of tryptophan absorption in the intestine of patients with Hartnup disease. A more generalized malabsorption of amino acids similar to that in the renal tubules was demonstrated subsequently by Scriver (12). Wong and Pillai (20) showed conclusively that intravenously administered tryptophan was normally metabolized. On the other hand, it was shown that a patient with congenital tryptophanuria had a defect in the kynurenine pathway of tryptophan metabolism (Fig. 1). However, the exact site of the enzymatic defect was not localized (16).

This paper describes the biochemical studies in a young patient who had marked cerebellar ataxia and photosensitive skin rash. This patient, however, differed clinically from patients with Hartnup disease and congenital tryptophanuria in that he was sensitive to oral nicotinic acid therapy.

\section{CASE STUDY}

This 7-year-old Caucasian boy was admitted to the University of Illinois Hospital for investigation because of ataxia and a skin rash.

He was delivered at term and in good condition after a normal pregnancy. His birth weight was $3.5 \mathrm{~kg}$ and there was no complication during the neonatal period. He started to smile at 2 months; grasp objects at 3 months; roll over at 5 months; sit up and say simple words at 8 months; stand up and crawl at 11 months; walk at 14 months; and talk in "sentences" at 2 years and 10 months. At 3 years of age, he was toilet-trained.

Since he started walking at 14 months of age, he was observed to be "clumsy," with poor coordination of the hands, poor balance in walking, and frequent falls. At the age of 2 years and 10 months, he was noted to "walk on his toes" and was inclined to lean forwards while running, falling frequently. At the age of 3 years, he was able to ride a tricycle without difficulty, although his hands were unsteady, he spilled food while feeding himself with a spoon, and he had a staggering gait. These symptoms and signs of ataxia disappeared completely at various times. The intermittent gross ataxia resulted in two falls which required visits to physicians for examination with suspicion of head injury. He had stuttering of speech and excessive drooling.

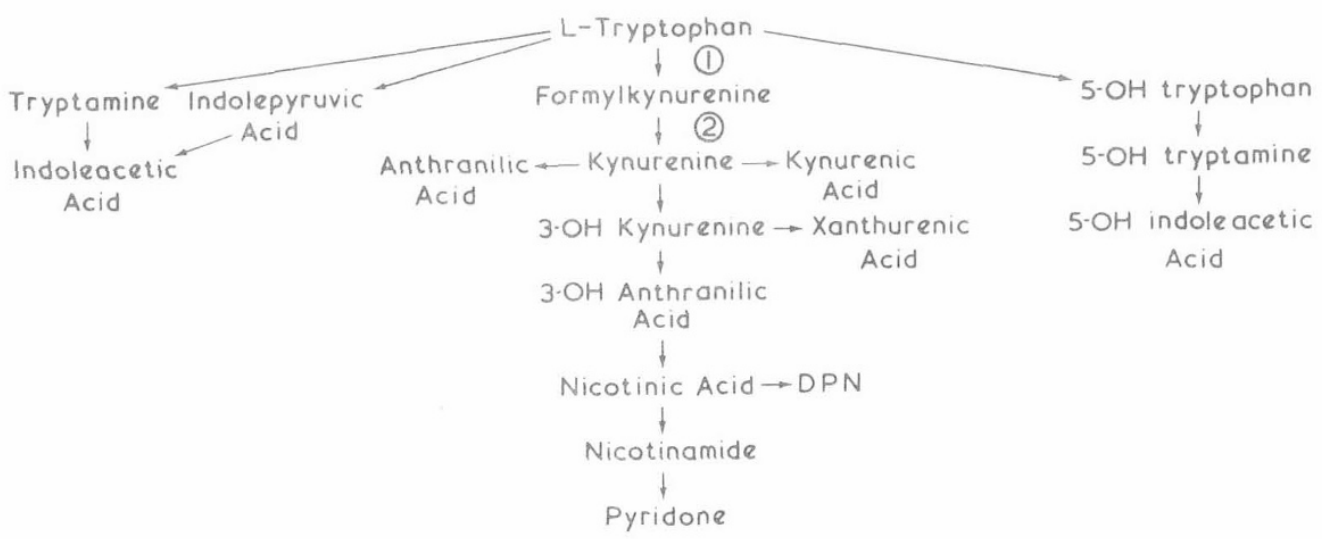

Fig. 1. Pathways of tryptophan metabolism. (1): tryptophan pyrrolase; (2): formylase. 
Since the age of 2 years, he had a dry, red, scaly rash on the face and the exposed areas of the arms and legs during the spring and the summer. Occasionally, blister formation was also observed. The parents quickly appreciated the association of exposure to sunlight and the appearance of the rash which would improve when he was well covered and disappeared completely during the winter. The possibility of dietary deficiency of niacin was excluded by a careful dietary history.

From the age of 5 years, he had symptoms of allergic rhinitis, and from the age of 7 years, he had a few attacks of bronchial asthma.

His father was an Irish-American and his mother an ItalianAmerican. Both parents, his 8-year-old brother, and his 6-year-old brother were healthy.

Physical examination at the age of 7 years showed that he was small for his age. His height, weight, and head circumference were $105 \mathrm{~cm}, 17.2 \mathrm{~kg}$, and $48 \mathrm{~cm}$, respectively, all of which were below the third percentile. His 6-year-old brother was taller as well as heavier.

There was a marked erythematous rash on the nasal bridge, the cheeks, the malar regions, the ears, and the exposed parts of the arms and legs. Scaling and peeling were prominent on the face. No abnormality was detected in the examination of the lungs, heart, and abdomen. His cranial nerves were intact. There was a mild increase of his muscular tone and spasticity of his lower limbs, but no muscular atrophy was found and muscular power was normal. Intentional tremor of upper and lower limbs was prominent but no nystagmus was observed. His gait was grossly ataxic and Romberg's sign was positive. Deep tendon reflexes were exaggerated but planter reflexes and sensory functions were normal.

Routine laboratory studies showed normal hemoglobin, white cell and differential count, serum electrolytes and blood urea nitrogen, serum glutamate-oxaloacetate and glutamate-pyruvate transaminases, lactate dehydrogenase, creatine phosphokinase, and alkaline phosphatase were within the normal range. Spina bifida occulta at the 5th lumbar vertebra was demonstrated in the $x$-ray of the spine. An audiogram and two electroencephalograms were entirely normal. Evaluation by a psychologist showed that his IQ was about 60 . Histologic examination of a skin biopsy showed changes of a chronic nonspecific dermatitis.

\section{METHODS}

Ion exchange chromatography (10) was used for the quantitation of amino acids. Tryptophan was identified by paper chromatography using Ehrlich's reagent (15) and was quantitated by the method of Denckla and Dewey (6). Paper chromatography (15) was used for the identification of tryptophan metabolites. The

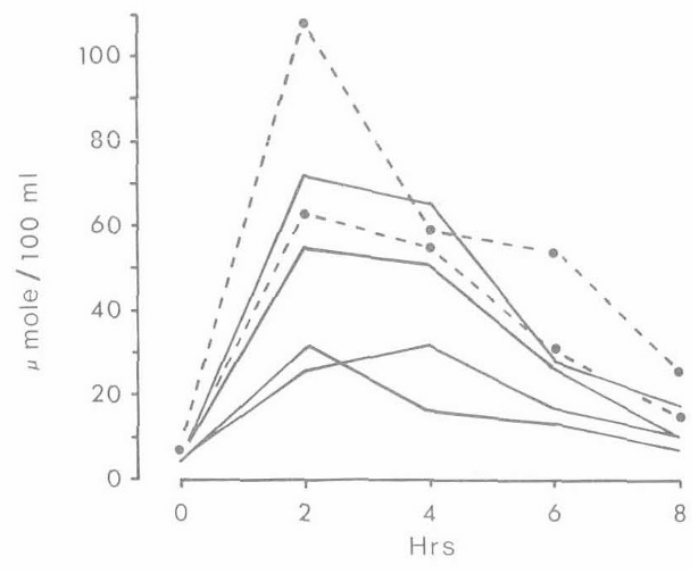

Fig. 2. Concentrations of plasma tryptophan. In this and in Figures 3 and 4 the solid lines (_- ) represent values for the control subjects and broken lines (- - ) represent those for the patient during tryptophan loading tests. methods of Coppini et al. (5), Carpenter and Kodicek (3) and Sharlit (13) were used to determine urinary kynurenine, $N^{\prime}$ methylnicotinamide, and indoxyl sulfate (indican), respectively. For the determination of indole-3-acetic acid. 5-hydroxyindoleacetic acid, and 5-hydroxytryptamine (serotonin), the procedures of Weissbach et al. (18), Udenfriend et al. (17), and Yuwiler et al. (21), respectively, were employed. The method of Chen (4) was adapted for the identification and estimation of formylkynurenine. A sensitive assay of tryptophan pyrrolase was developed by modifying the method of Knox and Mehler (8), using labeled tryptophan and 1-3 mg rat liver protein. Leukocytes were isolated from heparinized blood with dextran (14). Skin fibroblasts were cultured by routine methods (11). Renal clearance of tryptophan was calculated as described previously (19). Oral tryptophan loading tests were performed in the patient and comparable control subjects (children without metabolic disorders) after overnight fasting, using $100 \mathrm{mg}$ L-tryptophan $/ \mathrm{kg}$ body weight as described previously (20). Venous blood was obtained at $0,2,4,6$, and $8 \mathrm{hr}$ for the determination of tryptophan. After tryptophan loading, urine was collected every $6 \mathrm{hr}$ for other chemical determinations. Urea was removed from urine samples by purified urease obtained from the Sigma Chemical Company by a modification of the method of Henry and Chiamori (7).

\section{RESULTS}

Several quantitative determinations of amino acids in 24-hr urine samples from the patient showed no evidence of hyperaminoaciduria. In addition, random urine samples did not contain excessive quantities of indoxyl sulfate, indicating that the patient did not have Hartnup disease.

The mean of three fasting plasma tryptophan determinations in the patient was $6.21 \pm 0.720 \mu \mathrm{mol} / 100 \mathrm{ml}$ and that in six con-

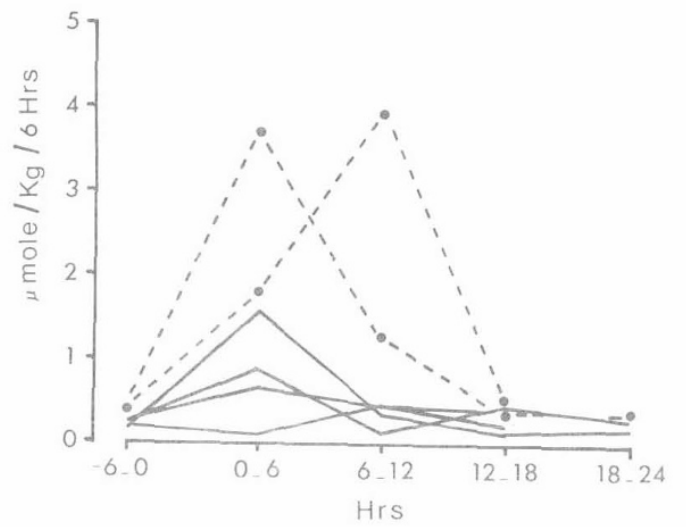

Fig. 3. Tryptophan excretion in urine; expressed as micromoles per $\mathrm{kg}$ per $6 \mathrm{hr}$.

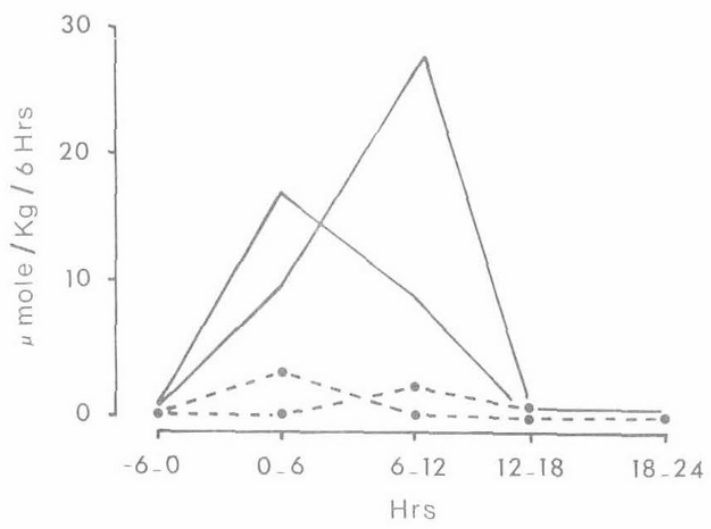

Fig. 4. Kynurenine excretion in urine; expressed as micromoles per $\mathrm{kg}$ per $6 \mathrm{hr}$. 


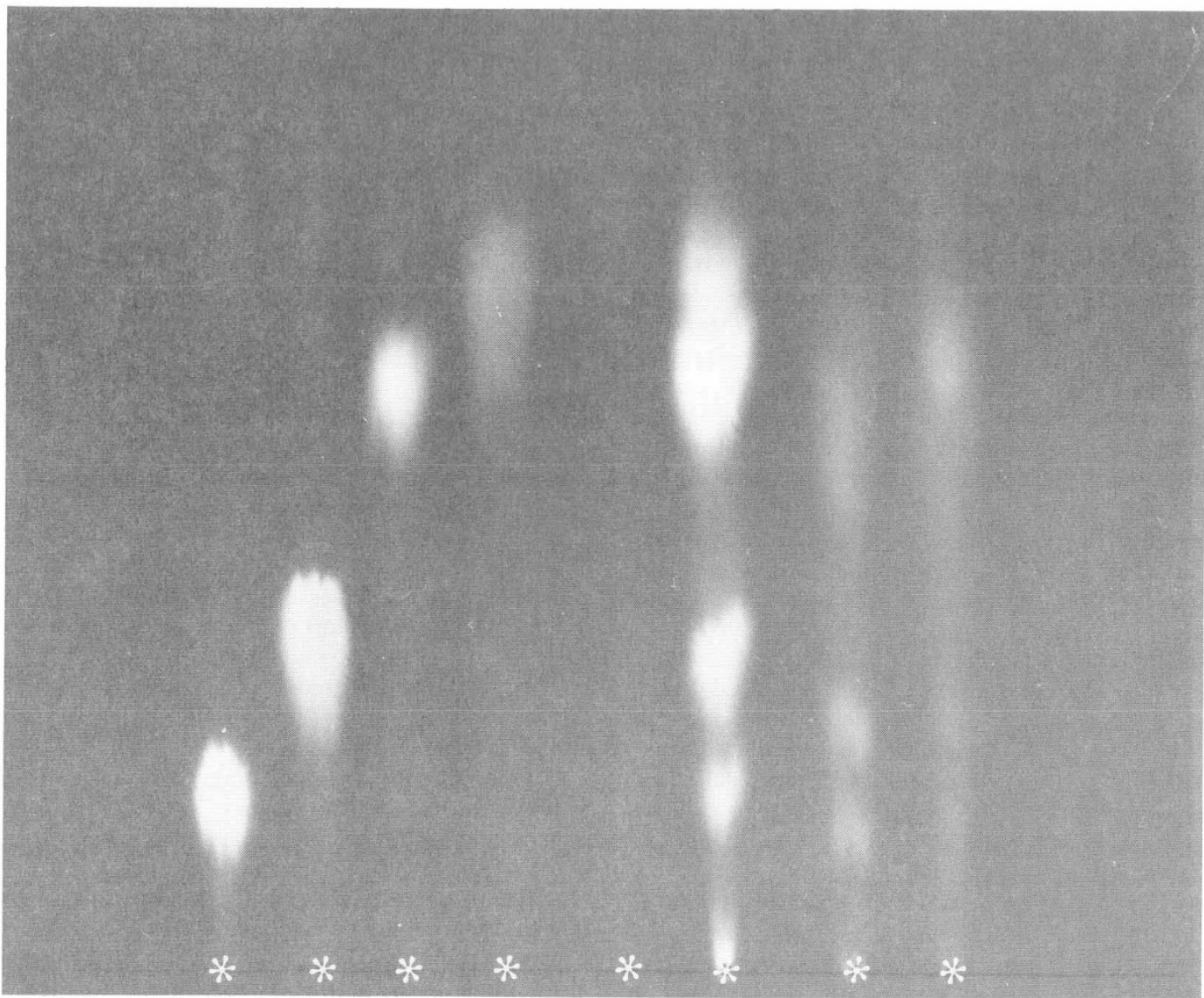

Fig. 5. One dimensional ascending paper chromatogram of urine under ultraviolet light. From left to right are samples of control urine with the following markers: xanthurenic acid, kynurenic acid, kynurenine, and formylkynurenine, respectively; 5th and 6th from the left are pre- and postloading urine samples, respectively, from a control subject; and 7th and 8 th from the left are pre- and postloading urine samples, respectively, from the patient.

trol subjects was $4.28 \pm 0.956 \mu \mathrm{mol} / 100 \mathrm{ml}$. There was no significant difference $(P>0.5)$. Plasma concentrations of tryptophan after tryptophan loading tests are shown in Figure 2. In contrast to patients with Hartnup disease (20), the levels of tryptophan at $0,2,4,6$, and $8 \mathrm{hr}$ in the patient increased markedly, suggesting that intestinal absorption was normal. In one loading test, there was a suggestion that tryptophan clearance in the plasma might be somewhat delayed in the patient.

Urinary excretion of tryptophan after loading tests is shown in Figure 3. From 0 to 6 and from 6 to $12 \mathrm{hr}$ after loading, the patient excreted more tryptophan than the control subjects. This may be a reflection of higher plasma tryptophan levels or increased renal loss. For this reason, renal clearance of tryptophan was calculated. Without tryptophan loading, the average renal clearance in the patient was $0.534 \mathrm{ml}$ plasma $/ 1.73 \mathrm{~m}^{2}$; that in three control subjects was $0.820 \mathrm{ml}$ plasma $/ 1.73 \mathrm{~m}^{2}$. The average renal clearance during the first $6 \mathrm{hr}$ of the loading tests was not different in the patient and the control subjects $(0.757 \mathrm{ml}$ and $0.706 \mathrm{ml}$, respectively). This indicated that there was no excessive renal loss of tryptophan in the patient.

Figure 4 shows the renal excretion of kynurenine after tryptophan loading. In contrast to the two control subjects, the patient showed little increase of kynurenine excretion despite the marked increase of plasma tryptophan.

Urinary excretion of $N^{\prime}$-methylnicotinamide in the patient after tryptophan loading was similar to that of the two control subjects (Table 1). It should be pointed out, however, that both the patient and the control subjects were given similar hospital diet for lunch and dinner after loading; the amount of exogenous nicotinamide from the diet was not quantitated.

The excretion of indoxyl sulfate after tryptophan loading in the patient was similar to that in two control subjects (Table 1). There was no marked or delayed increase, characteristic of Hartnup
Table 1. Urinary tryptophan metabolites

\begin{tabular}{|c|c|c|c|c|c|c|}
\hline & \multicolumn{5}{|c|}{ Hours } & \multirow{2}{*}{$\begin{array}{r}\text { Total, } \\
-\quad 0-24 \\
2 \quad \mathrm{hr}\end{array}$} \\
\hline & $-6-0^{1}$ & $0-6^{2}$ & $6-12^{2}$ & $12-18^{2}$ & $18-24^{2}$ & \\
\hline \multicolumn{7}{|c|}{$\begin{array}{l}N^{1} \text {-Methylnicotina- } \\
\text { mide }\end{array}$} \\
\hline$(\mathrm{Cl})$ & 0.41 & 0.70 & 1.51 & 1.16 & 0.93 & 4.30 \\
\hline (C2) & 0.52 & 0.84 & 1.44 & 0.67 & 1.63 & 4.58 \\
\hline (P) & 0.17 & 0.26 & 1.33 & 0.64 & 1.33 & 3.56 \\
\hline (P) & 0.19 & 0.90 & 0.78 & 0.80 & 0.75 & 3.23 \\
\hline \multicolumn{7}{|c|}{ Indoxyl sulfate } \\
\hline$(\mathrm{Cl})$ & 1.98 & 1.43 & 1.88 & 0.92 & 1.23 & 5.46 \\
\hline (C2) & 2.40 & 2.27 & 2.00 & 2.47 & 1.35 & 8.09 \\
\hline$(\mathrm{P})$ & 1.59 & 1.39 & 3.58 & 1.11 & 3.26 & 9.34 \\
\hline (P) & 1.90 & 1.71 & 2.98 & 3.58 & 2.10 & 10.37 \\
\hline \multicolumn{7}{|c|}{$\begin{array}{l}\text { Indole-3-acetic } \\
\text { acid }\end{array}$} \\
\hline$(\mathrm{Cl})$ & 0.02 & 0.54 & 0.44 & 0.21 & 0.01 & 1.20 \\
\hline (C2) & 0.03 & 0.82 & 0.10 & 0.09 & 0.03 & 1.04 \\
\hline (P) & 0.02 & 1.31 & 0.18 & 0.05 & 0.03 & 1.57 \\
\hline (P) & 0.03 & 0.71 & 1.03 & 0.01 & 0.01 & 1.76 \\
\hline \multicolumn{7}{|c|}{$\begin{array}{c}\text { 5-Hydroxyindole- } \\
\text { acetic acid }\end{array}$} \\
\hline$(\mathrm{Cl})$ & 0.12 & 0.17 & 0.10 & 0.10 & 0.11 & 0.48 \\
\hline (C2) & 0.20 & 0.34 & 0.18 & 0.34 & 0.10 & 0.96 \\
\hline$(\mathrm{P})$ & 0.18 & 0.10 & 0.30 & 0.12 & 0.18 & 0.70 \\
\hline (P) & 0.15 & 0.33 & 0.28 & 0.26 & 0.12 & 0.99 \\
\hline
\end{tabular}

${ }^{1}$ C: control subject; P: patient,

${ }^{2}$ All values are expressed as micromoles per $\mathrm{kg}$ per $6 \mathrm{hr}$.

${ }^{3}$ All values are expressed as micromoles per kg per $24 \mathrm{hr}$. 
disease (20). In the same manner, the excretion of indole-3-acetic acid and 5-hydroxyindole acetic acid in the patient was similar to that in the control subjects (Table 1).

Urinary excretion of fluorescent metabolites after tryptophan loading tests is demonstrated in Figure 5. Using equivalent amounts of urine based on creatinine concentrations, multiple runs of paper chromatography examined under ultraviolet light showed similar results in that the patient failed to show a significant increase of urinary xanthurenic acid, kynurenic acid, kynurenine, and formylkynurenine. To further identify formylkynurenine, urine samples were subjected to two-dimensional paper chromatography using various solvent systems and Ehrlich's reagent for staining. In all systems used, formylkynurenine could not be separated from the large amounts of urea. Therefore, urea was removed from the urine samples by incubation with purified urease. The result is demonstrated in Figures 6 and 7. It is seen that from 0 to 6 and from 6 to $12 \mathrm{hr}$ after tryptophan loading, a control subject excreted large amounts of formylkynurenine and kynurenine (Fig. 6). On the other hand, the patient failed to excrete significant amounts of these substances at any time after tryptophan loading (Fig. 7).

Using L- $\left[{ }^{14} \mathrm{C}\right]$ methylene tryptophan as substrate and $1-3 \mathrm{mg}$ rat liver protein, $1.6-3.5 \mathrm{nmol}$ kynurenine were produced $/ \mathrm{hr} / \mathrm{mg}$ protein. However, using 3-4 mg white blood cell protein (approximately $50-60 \times 10^{6}$ cells) and $7-8 \mathrm{mg}$ fibroblast protein (approximately 8 confluent flasks with surface area of $75 \mathrm{~cm}^{2}$ each) from normal subjects, no activity of tryptophan pyrrolase was detected on several occasions. Activity as little as $3-5 \%$ of that found in rat liver should be detectable by this method.

Treatment with $100 \mathrm{mg}, 50 \mathrm{mg}$, and $10 \mathrm{mg}$ nicotinic acid in the patient resulted in flushing, "burning" sensation, and "itching" of skin. On the other hand, treatment with $50 \mathrm{mg}$ or $100 \mathrm{mg}$ of nicotinamide did not produce any untoward effect, but the photosensitive, pellagra-like skin rash did not appear during prolonged exposure to sunlight while the patient was in a summer camp. However, there was no change in the neurologic abnormalities.

Because of the possibility of an interaction between nicotinic acid and serotonin, blood concentration of serotonin was determined on two occasions without any medication or tryptophan loading. The patient had 0.32 and $0.30 \mu \mathrm{g}$ serotonin $/ \mathrm{ml}$ blood and three control subjects had $0.20,0.31$, and $0.27 \mu \mathrm{g} / \mathrm{ml}$, respectively. These results are similar to reported values. There was no evidence of increased serotonin in the blood of the patient.

\section{DISCUSSION}

Hartnup disease is characterized biochemically by hyperaminoaciduria, excessive indicanuria, and malabsorption of tryptophan (9). The latter may be demonstrated by a lack of increase or small increase in plasma tryptophan after an oral tryptophan loading test (20). The decrease of kynurenine and $N^{\prime}$-methylnicotinamide excretion and the excessive increase of indole-3-acetic acid and indoxyl sulfate (indican) excretion may also be explained on the basis of tryptophan malabsorption (1). On the other hand, intravenously administered tryptophan is normally metabolized (20), indicating that there is no defect in the intracellular metabolism of tryptophan.

In our patient, intestinal malabsorption of tryptophan can be excluded by the marked increase of plasma tryptophan after oral tryptophan loading and by the lack of excessive excretion of indoxyl sulfate and 5-hydroxyindole acetic acid. In addition, the absence of hyperaminoaciduria and the normal renal clearance of tryptophan effectively exclude Hartnup disease in this patient. Therefore, the decreased excretion of kynurenine requires a different explanation. After tryptophan loading, the absence of

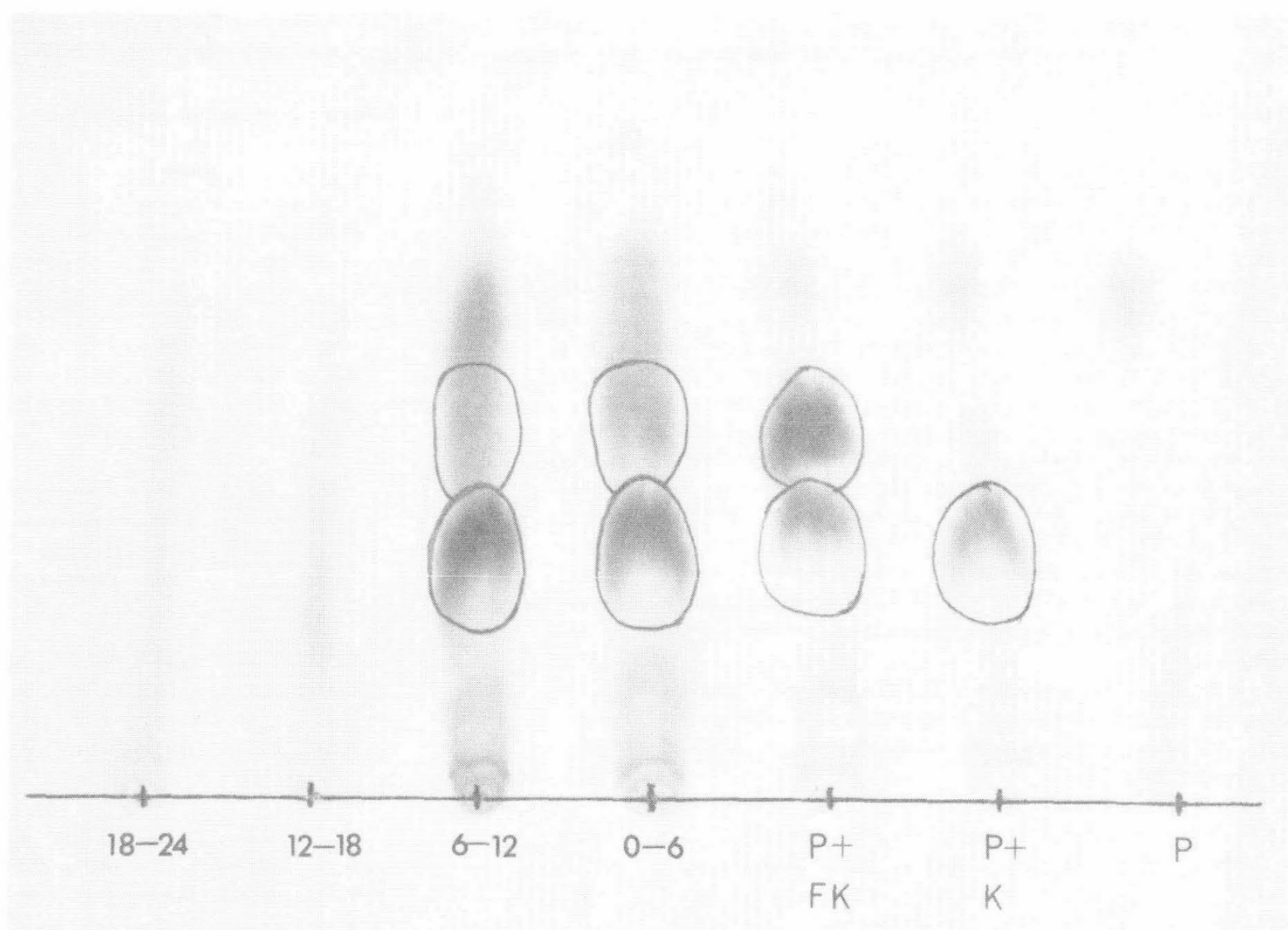

Fig. 6. One-dimensional ascending paper chromatography of urine samples stained with Ehrlich's reagent from a control subject. $P$ : preloading urine; $P+K$ : preloading urine plus kynurenine marker; $P+F K$ : preloading urine plus formylkynurenine and kynurenine markers; $0-6,6-12,12-18,18-24$ : urine samples obtained after tryptophan loading for the respective periods. 


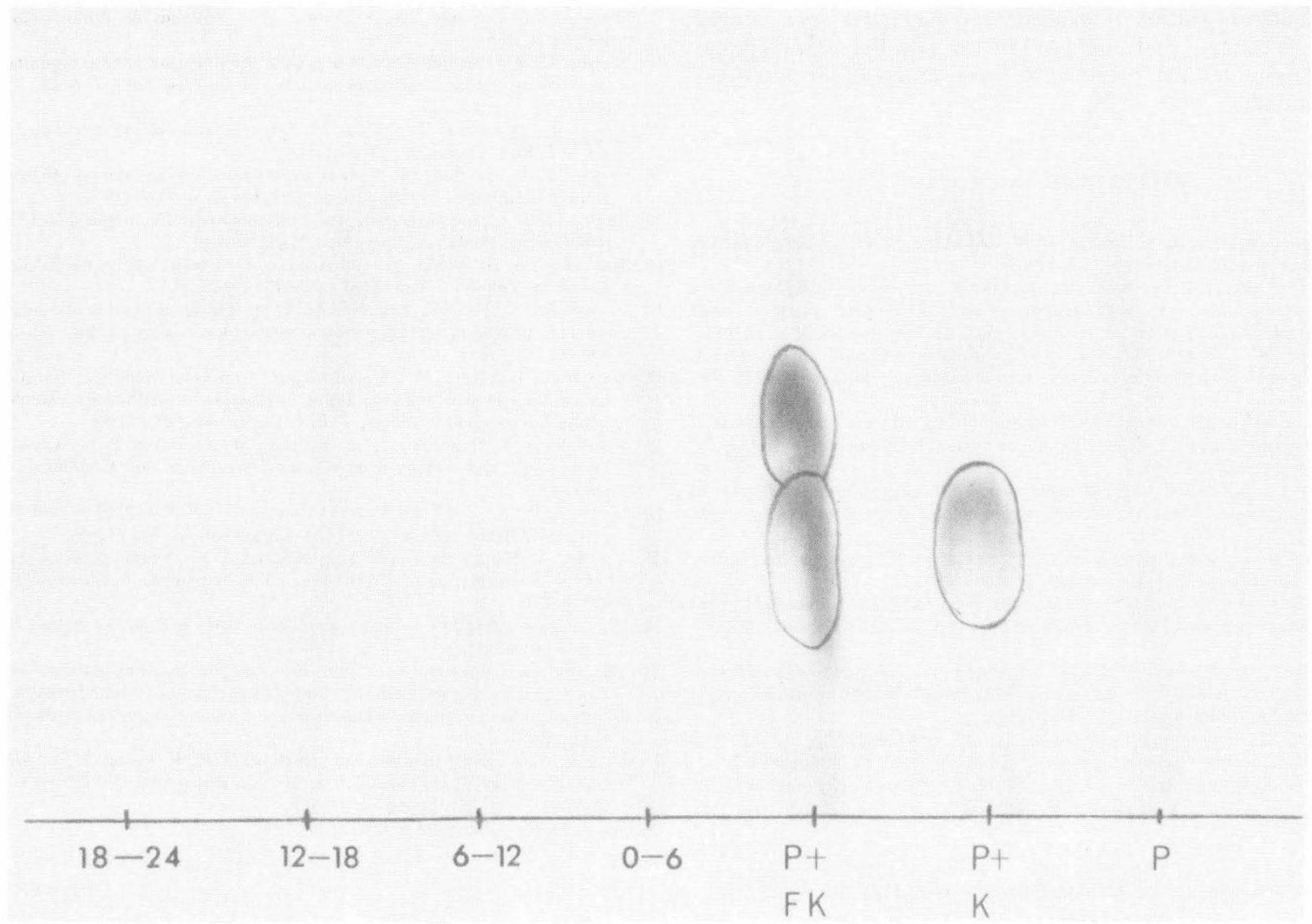

Fig. 7. One-dimensional ascending paper chromatography of urine samples stained with Ehrlich's reagent from the patient. Symbols are the same as those in Figure 6.

increase in the excretion of tryptophan metabolites in the kynurenine pathway, such as kynurenine, xanthurenic acid, and kynurenic acid, suggests a metabolic block in tryptophan oxidation.

In 1963, Tada and his coworkers (16) studied a patient with congenital tryptophanuria and dwarfism. This disorder was characterized clinically by mental retardation, photosensitive pellagralike skin rash, cerebellar ataxia, and dwarfism. Biochemically, the patient excreted increased amounts of tryptophan and decreased amounts of kynurenine and $N^{\prime}$-methylnicotinamide with and without tryptophan loading. In addition, there was a delayed clearance of plasma tryptophan after an oral tryptophan loading test. A defect in the conversion of tryptophan to kynurenine was postulated; however, the site of the metabolic block was not localized (Fig. 1).

In many respects, our patient is very similar to that of Tada et al. (16). However, there are clinical differences. In our patient, there are signs of upper motor neuron dysfunction such as increased muscular tone, spasticity, and exaggerated deep tendon reflexes. In addition, the administration of $40 \mathrm{mg}$ nicotinic acid $/ \mathrm{kg}$ body weight (total $480 \mathrm{mg}$ ) in the patient of Tada et al. did not produce any untoward effect. This is similar to the observations in Hartnup disease (19) but is in contrast to our patient.

Although confirmation of the enzymatic defect in our patient requires a liver biopsy, the results of the two tryptophan loading tests strongly suggest a metabolic block in the kynurenine pathway of tryptophan metabolism. The lack of excretion of formylkynurenine, kynurenine, and other metabolites in the same pathway after tryptophan loading suggests that the metabolic block is at the step catalyzed by tryptophan pyrrolase (Fig. 1). In addition, other possible mechanisms of kynurenine deficiency may be confidently excluded. First, if the primary defect is due to excessive renal loss of tryptophan, it may be expected that plasma tryptophan levels before and during tryptophan loading would be subnormal and that the renal clearance of tryptophan would be unduly high.
Second, if the primary defect is due to increased catabolism of formylkynurenin: and kynurenine, excretion of other metabolites in the same pithway may be expected to be increased. The occurrence of signs and symptoms compatible with nicotinamide deficiency also tends to render this possibility unlikely.

The lack of marked hypertryptophanemia under normal conditions and during tryptophan loading tests in our patient cannot be readily explained. It seems possible that the ingested tryptophan may be "siphoned off" via some unknown or normally minor pathway or pathways. This possibility may be tested by tracing labeled tryptophan in vivo. However, administering radioactive tryptophan to this patient was considered unjustified.

The mechanism for the unusual "sensitivity" to oral nicotinic acid in our patient is unknown. Determination of whether this is due to the lack of formylkynurenine and is therefore different from the patient of Tada et al. (16) requires further observation.

The clinical signs and symptoms in our patient seemed to be more severe than those observed in patients with Hartnup disease. The upper motor neuron abnormalities were persistent throughout the period of observation and the cerebellar ataxia was more profound and longer lasting. This suggests that the deficiency of tryptophan metabolites was more marked because of an enzymatic block in our patient than that in patients with Hartnup disease with a defect in tryptophan absorption which, nevertheless, allows some dietary tryptophan to be absorbed.

\section{SUMMARY}

A patient with photosensitive, pellagra-like skin rash, reversible cerebellar ataxia, upper motor neuron dysfunction, and mental and physical retardation was studied using oral tryptophan loading tests. The results suggested that there was a defect at the first step of tryptophan metabolism in the kynurenine pathway, leading to a deficiency of formylkynurenine, kynurenine, and other metabo- 
lites. Oral administration of nicotinic acid in small doses produced flushing, "burning," and "itching" of the skin but oral nicotinamide was well tolerated, resulting in improvement of the photosensitive skin rash.

\section{REFERENCES AND NOTES}

1. Asatoor, A. M., Craske, J., London, D. R., and Milne, M. D.: Indole production in Hartnup disease. Lancet, $i: 126$ (1963).

2. Baron, D. N., Dent, C. E., Harris, H., Hart, E. W., and Jepson, J. B.: Hereditary pellagra-like skin rash with temporary cerebellar ataxia, constant renal aminoaciduria, and other bizarre biochemical features. Lancet, ii: 421 (1956).

3. Carpenter, K. J., and Kodicek, E.: The fluorimetric estimation of $N^{\prime}$-methylnicotinamide and its differentiation from coenzyme I. Biochemistry, 46: 421 (1950).

4. Chen, J., and Matchett, W. H.: Occurrence of $\mathrm{N}$-formylkynurenine in extracts of Neurospora crassa: Evidence for the activity of tryptophan pyrrolase. J. Bacteriol., 118: 837 (1974).

5. Coppini, D., Benassi, C. A., and Montorsi, M.: Quantitative determination of tryptophan metabolites (via kynurenine) in biologic fluids. Clin. Chem., 5: 391 (1959).

6. Denckla, W. D., and Dewey, K.: The determination of tryptophan in plasma, liver, and urine. J. Lab. Clin. Med., 69: 160 (1967).

7. Henry, R. J., and Chiamori, N.: On the direct Nesslerization of ammonia formed by urease treatment of blood, serum and urines. Amer. J. Clin. Pathol., 29: 277 (1958).

8. Knox, W. E., and Mehler, A. H.: The conversion of tryptophan to kynurenine in liver. I. The coupled tryptophan peroxidase-oxidase system forming formylkynurenine. J. Biol. Chem., 187: 419 (1950).

9. Milne, M. D., Crawford, M. A., Girão, C. B., and Loughridge, L. W.: The metabolic disorder in Hartnup disease. Quart. J. Med., 29: 407 (1960).

10. Moore, S., Spackman, D. H., and Stein, W. H.: Chromatography of amino acids on sulfonated polystyrene resins: An improved system. Anal. Chem., 30: 1185 (1958).

Copyright (c) 1976 International Pediatric Research Foundation, Inc.
11. Paul, J.: In: Cell and Tissue Culture (The Williams \& Wilkins Co., Inc., Baltimore, 1961).

12. Scriver, C. R.: Hartnup disease, a genetic modification of intestinal and renal transport of certain neutral alpha-amino acids. New Engl. J. Med., 273: 530 (1965).

13. Sharlit, H.: A method for the quantitative estimation of indoxyl compounds in urine. J. Biol. Chem., 99: 537 (1933)

14. Skoog, W. A., and Beck, W. S.: Studies on fibrinogen, dextran and phytohemagglutinin methods of isolating leukocytes. Blood, 11:436 (1956).

15. Smith, I.: In: Chromatographic and Electrophoretic Techniques, Vol. 1, p. 96 (Interscience Publishers, Inc.. New York, 1960).

16. Tada, K., Ito, H., Wada, Y., and Arkawa, T.: Congenital tryptophanuria with dwarfism. Tokohu J. Exp. Med., 80: 118 (1963).

17. Udenfriend, S., Titus, E., and Weissbach, H.: The identification of 5-hydroxy-3indoleacetic acid in normal urine and method for its assay. J. Biol. Chem., 216: 499 (1955).

18. Weissbach, H., King, W., Sjoerdsma, A., and Udenfriend, S.: Formation of indole-3-acetic acid and tryptamine in animals: A method for estimation of indole-3-acetic acid in tissues. J. Biol. Chem., 234: 81 (1959).

19. Wong, P. W. K., Lambert, A. M., Pillai, P. M. and Jones, P. M.: Observations on nicotinic acid therapy in Hartnup disease. Arch. Dis. Childhood, 42: 642 (1967).

20. Wong, P. W. K., and Pillai, P. M : Clinical and biochemical observations in two cases of Hartnup disease. Arch. Dis. Childhood, 4I: 383 (1966).

21. Yuwiler, A., Plotkin, S., Geller, E., and Ritvo, E. R .: A rapid accurate procedure for the determination of serotonin in whole human blood. Biochem. Med., 3: 426 (1970).

22. The authors would like to thank Kathryne Curley and Nancy Becker for their technical assistance.

23. This study was supported by a grant from the National Foundation-March of Dimes and by a grant from the Illinois Department of Mental Health.

24. Informed consent was obtained according to a protocol approved by the Research Committee.

25. Requests for reprints should be addressed to: P. W. K. Wong, M.D., Section of Genetics, Department of Pediatrics, Rush Medical School, 1753 W. Congress Parkway, Chicago, III. 60612 (USA).

26. Accepted for publication February 17, 1976.

\title{
Effect of Postweanling Pyridoxine Deficiency on Growth and Concentration of the Coenzyme Pyridoxal-5'-phosphate in Heart, Kidneys, Lungs, and Adrenals in Rats
}

\author{
HEMMIGE N. BHAGAVAN, ${ }^{34}$ JOHN M. KOOGLER, JR., AND DAVID B. COURSIN
}

Research Institute, St. Joseph Hospital, Lancaster, Pennsylvania, USA

\section{Extract}

Dietary pyridoxine deficiency induced in postweanling rats led to severe growth retardation and growth failure, with a sharp increase in the mortality rate after about 8 weeks on the diet. The absolute weights of heart, lungs, kidneys, and adrenals were all lower in the deficient animals than those of the controls. However, when the weights were expressed in terms of percentage of body weight, a 2 -fold increase was observed indicative of marked hypertrophy. The depletion of the coenzyme pyridoxal-5'-phosphate from these tissues was extensive (heart $48 \%$, lungs $85 \%$, and kidneys and adrenals $88 \%$ ). The extent of loss of the coenzyme from the tissues suggests possible functional changes.

\section{Speculation}

Functional disturbances may be expected as a consequence of the marked hypertrophy and the extensive depletion of the coenzyme pyridoxal-5'-phosphate from the heart, lungs, kidneys, and adrenals in postweanling pyridoxine-deficient rats. 